\title{
OBTENCIÓN DE AZÚCARES FERMENTABLES A PARTIR DE ASERRÍN DE PINO PRETRATADO SECUENCIALMENTE CON ÁCIDO-BASE
}

\author{
María Daniela RODRÍGUEZ*, María Lorena CASTRILLO, Juan Ernesto VELÁZQUEZ, \\ Gustavo Raúl KRAMER, Cyntia SEDLER, Pedro Darío ZAPATA y Laura VILLALBA
}

Laboratorio de Biotecnología Molecular, Instituto de Biotecnología de Misiones - Facultad de Ciencias Exactas, Químicas y Naturales, Universidad Nacional de Misiones. Ruta Nacional 12, kilómetro 7.5, Posadas, Misiones, Argentina, C. P. 3300

*Autor para correspondencia: daniela_1305@yahoo.com.ar

(Recibido enero 2016; aceptado agosto 2016)

Palabras clave: residuo lignocelulósico, diseño factorial, lignina, sacarificación.

\section{RESUMEN}

El uso de residuos lignocelulósicos constituye una fuente abundante y de bajo costo, que genera, además, una solución al problema de su disposición final. El objetivo del trabajo fue evaluar el efecto de distintas condiciones de pretratamiento secuencial ácido-base de aserrín de pino, sobre la liberación de azúcares reductores en la etapa de sacarificación. El material lignocelulósico se pretrató en dos etapas: la primera, se realizó con ácido sulfúrico (PT1) y la segunda con hidróxido de sodio (PT2), ambas con tres niveles: 1,2 y $3 \%$ mediante un diseño $3^{2}$ factorial. La hidrólisis enzimática se realizó a $50^{\circ} \mathrm{C}$ y $150 \mathrm{rpm}$ en frascos de $50 \mathrm{~mL}$. Se tomaron muestras a las $0.15,24$, 48, 72 y 96 h. Se determinó el porcentaje de lignina ácido soluble y la concentración de azúcares reductores. El nivel óptimo de cada factor que favoreció la mayor deslignificación correspondió a $3 \%$ de ácido sulfúrico y $3 \%$ de hidróxido de sodio. La producción de azúcares reductores no se vio afectada por el PT2, pero sí por el PT1, alcanzando un máximo para el nivel 0 del mismo ( $2 \%$ de ácido sulfúrico). Se optimizó el pretratamiento secuencial ácido-base y se comprobó además que éste mejora la hidrólisis enzimática respecto al aserrín de pino sin pretratar. La cantidad de azúcares reductores obtenidos fue comparable a la obtenida por otros autores.

Key words: lignocellulosic waste, factorial design, lignin, saccharification.

\begin{abstract}
The use of lignocellulosic waste constitutes an abundant and inexpensive source; it also offers a solution to the problem of waste disposal. The aim of the study was to evaluate the effect of different conditions of a sequential acid-base pretreatment of pine sawdust, through the release of reducing sugars in the saccharification step. The lignocellulosic material was pretreated in two stages: acid pretreatment (PT1), using sulfuric acid and basic pretreatment (PT2), employing sodium hydroxide at three levels each one: 1, 2 and $3 \%$ in a factorial design. Enzymatic hydrolysis was carried out at $50{ }^{\circ} \mathrm{C}$ and 150 $\mathrm{rpm}$ in $50 \mathrm{~mL}$ vials. Samples were taken at $0.15,24,48,72$ and $96 \mathrm{~h}$. The percentage of acid soluble lignin and the concentration of reducing sugars were determined. The
\end{abstract}


optimum level of each factor favoring the highest delignification corresponded to $3 \%$ sulfuric acid and $3 \%$ sodium hydroxide. The production of reducing sugars was not affected by the PT2, but by the PT1, achieving a maximum for level 0 thereof (2 $\%$ of sulfuric acid). The sequential acid-base pretreatment was optimized and it was found that this pretreatment improves enzymatic hydrolysis of pine sawdust respect to unpretreated samples. The amount of reducing sugars obtained was comparable to that obtained by other authors.

\section{INTRODUCCIÓN}

La lignocelulosa es el componente principal de la biomasa y cerca de la mitad de la materia producida por la fotosíntesis. Se compone de tres tipos de polímeros: celulosa, hemicelulosa y lignina, que están estrechamente entrelazados y químicamente unidos por fuerzas no covalentes y mediante entrecruzamientos covalentes (Pérez et al. 2002). En este contexto, el uso de residuos lignocelulósicos constituye una fuente abundante y de bajo costo, que genera además una solución al problema de su disposición final (Lin y Tanaka 2006, López-Miranda et al. 2009). La provincia de Misiones (Argentina) está ubicada en una región cuya principal actividad económica es la forestal, por lo que la generación de residuos agroforestales crea un problema ambiental que es necesario resolver (Romero 2012, Mantulak et al. 2013).

El bioetanol de segunda generación producido a partir de diferentes materiales lignocelulósicos como la madera, tiene el potencial de sustituir o complementar a los combustibles fósiles utilizados actualmente. El proceso para la conversión de lignocelulosa a etanol requiere la deslignificación de la biomasa lignocelulósica, para liberar a la celulosa de la hemicelulosa y de la lignina, presentes en el complejo celulosa-hemicelulosa-lignina y lograr así, la despolimerización de los hidratos de carbono para producir azúcares simples y la fermentación de hexosas y pentosas para producir etanol. Entre los procesos descritos anteriormente, la deslignificación de las materias primas lignocelulósicas es una de las etapas más difícil de resolver a bajo costo (Lin y Tanaka 2006). Un pretratamiento efectivo debería lograr una buena recuperación de todos los carbohidratos, aumentar la digestibilidad de la celulosa en la subsecuente hidrólisis enzimática, minimizar la generación de productos derivados de la degradación de azúcares y de lignina, ser de bajo costo y fácilmente operable. Un gran número de pretratamientos diferentes han sido sugeridos en las últimas décadas y pueden ser divididos en distintas categorías: físicos, químicos, biológicos o una combinación de éstos (Brodeur et al. 2011).

El pretratamiento de la biomasa lignocelulósica con ácido diluido es uno de los métodos más eficaces que afectan predominantemente la hemicelulosa, con poco impacto en la degradación de la lignina (Silverstein et al. 2007). Por otro lado, los pretratamientos alcalinos son más efectivos en disolver la lignina, mostrando una menor solubilización de la celulosa y la hemicelulosa. En la hidrólisis alcalina, el álcali induce el hinchamiento de la celulosa, lo que lleva a un incremento del área interna superficial, dejando a la celulosa más accesible al ataque enzimático (Carvalheiro et al. 2008). Se ha encontrado que el pretratamiento secuencial ácido-base tiene un efecto positivo en los rendimientos de azúcar producidos durante la hidrólisis enzimática, mejorando estos rendimientos en comparación con los obtenidos con una sola etapa de pretratamiento (ácida o alcalina) (Sanchez et al. 2015).

El objetivo del trabajo fue evaluar el efecto de distintas concentraciones de ácido-base, durante el pretratamiento secuencial de aserrín de pino, sobre la liberación de azúcares reductores.

\section{MATERIALES Y MÉTODOS}

\section{Materia prima}

El aserrín de Pinus sp. se recolectó del aserradero Valerio Oliva S.A. ubicado en la ciudad de Garupá (Misiones-Argentina) y se secó a temperatura ambiente hasta un contenido de humedad promedio de $18.6 \%$.

\section{Pretratamiento}

El material lignocelulósico se pretrató en matraces Erlenmeyer de $500 \mathrm{~mL}$ con $40 \mathrm{~g}$ (peso seco), en dos etapas: pretratamiento ácido (PT1) y pretratamiento básico (PT2). E1 PT1 consistió en la adición de ácido sulfúrico en una relación sólido/líquido $1: 5(\mathrm{~m} / \mathrm{v})$ y el calentamiento en autoclave a $121{ }^{\circ} \mathrm{C}$ y $1 \mathrm{~atm}$ de presión durante $60 \mathrm{~min}$. El material se filtró, se lavó con agua potable varias veces hasta que las aguas de 
lavado alcanzaron un pH 6. Posteriormente se lavó con agua destilada, se extrudió y secó a temperatura ambiente. Se realizó el PT2, en $30 \mathrm{~g}$ del material resultante, utilizando hidróxido de sodio en una relación sólido/líquido de 1:6 (m/v). El calentamiento se realizó en autoclave a $121{ }^{\circ} \mathrm{C}$ y 1 atm de presión durante $90 \mathrm{~min}$. El material se filtró, se lavó con agua potable hasta que las aguas de lavado alcanzaron $\mathrm{pH} 6$, luego se lavó con agua destilada, se extrudió y secó a temperatura ambiente. Se obtuvo un promedio de $23 \mathrm{~g}$ (peso seco) de material al finalizar el PT2.

El esquema de trabajo para cada combinación de pretratamiento se muestra en la figura 1.

\section{Sacarificación}

Se pesaron $0.29 \mathrm{~g}$ (peso seco) de aserrín pretratado, se mezclaron con $14.5 \mathrm{~mL}$ de sustancia amortiguadora de acetato de sodio $0.05 \mathrm{M}(\mathrm{pH} 4.8)$ y 20 $\mathrm{U} / \mathrm{g}$ celulosa $(50 \mu \mathrm{L})$ de Celluclast 1.5 L (Sigma). La hidrólisis enzimática del aserrín pretratado se realizó a $50{ }^{\circ} \mathrm{C}$ y $150 \mathrm{rpm}$ en frascos de $50 \mathrm{~mL}$ (Fig. 1). Se tomaron muestras durante $0.15,24,48,72$ y $96 \mathrm{~h} y$ se utilizó aserrín de pino sin pretratar como testigo negativo del proceso, exponiéndolo a las mismas condiciones de hidrólisis que el aserrín pretratado.

\section{Análisis}

Se determinó el contenido de sólidos totales del aserrín sin pretratar y pretratado, de acuerdo con el protocolo estándar del NREL/TP-510-42621

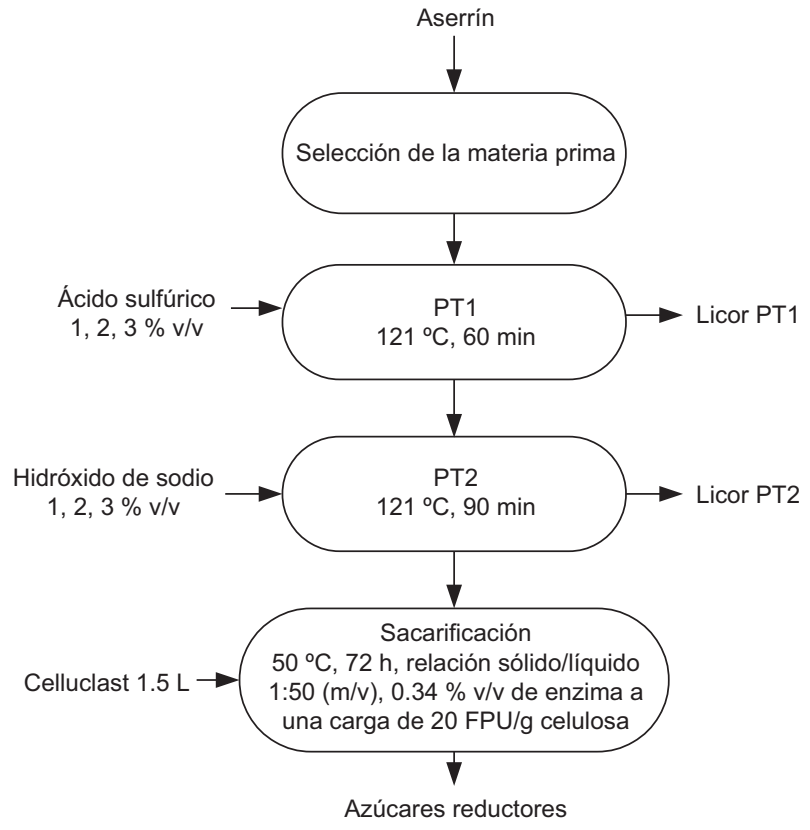

Fig. 1. Diagrama de flujo de las etapas de pretratamiento (PT1 y PT2) y sacarificación
(NREL 2008d). El contenido de extractivos se determinó mediante NREL/TP-510-42619 (NREL 2008a), el porcentaje de lignina soluble en ácido (\% ASL, por sus siglas en inglés) y lignina insoluble en ácido (\% AIL, por sus siglas en inglés) se determinó según NREL/TP-510-42618 (NREL 2011) y el contenido de cenizas según NREL/TP-510-42622 (NREL 2008c). El contenido de carbohidratos totales se midió calculando la diferencia entre el $100 \%$ y la suma de porcentajes de humedad, extraíbles, cenizas y lignina (López-Miranda et al. 2009). Los azúcares reductores se midieron de acuerdo con el método del ácido dinitrosalicílico (DNS) (Miller 1959).

La actividad enzimática (FPA, por sus siglas en inglés) se realizó mediante la técnica NREL/ TP-510-42628 (NREL 2008b), para ello se utilizaron $10 \mathrm{mg}$ de papel filtro Whatman No. 1 como sustrato y $0.1 \mathrm{~mL}$ de extracto enzimático diluido en amortiguador de acetato de sodio $0.05 \mathrm{M}(\mathrm{pH}$ 4.8) a $50^{\circ} \mathrm{C}$ durante $60 \mathrm{~min}$ (una unidad de actividad enzimática fue definida como la cantidad de enzima requerida para liberar $1 \mu \mathrm{mol} / \mathrm{mL}$ min de glucosa bajo las condiciones ensayadas).

\section{Estadística}

Las variables estudiadas fueron las concentraciones de ácido y álcali en los procesos de pretratamiento secuencial PT1 y PT2, respectivamente utilizando tres niveles para cada una de ellas $(1,2$ y $3 \% \mathrm{v} / \mathrm{v})$, mediante un diseño $3^{2}$ factorial.

Para establecer si existían diferencias significativas entre los pretratamientos, el testigo y el tiempo de sacarificación, se realizó un análisis de varianza con un nivel de confianza del $95 \%$. El análisis estadístico se realizó con el programa Statgraphics Centurion XV versión 15.2.05.

\section{RESULTADOS}

\section{Pretratamiento}

En el cuadro I se muestra la composición química del aserrín de pino utilizado.

CUADRO I. COMPOSICIÓN QUÍMICA DELASERRÍN DE PINO

\begin{tabular}{lc}
\hline Componente & $\%$ del peso seco total $(\mathrm{m} / \mathrm{m})$ \\
\hline Carbohidratos & $65.17 \pm 0.74$ \\
Lignina insoluble en ácido & $23.99 \pm 1.28$ \\
Lignina soluble en ácido & $0.46 \pm 0.03$ \\
Extractivos & $1.82 \pm 0.29$ \\
Cenizas & $0.59 \pm 0.01$ \\
\hline
\end{tabular}


CUADRO II. DISEÑO FACTORIAL. PRETRATAMIENTO CON ÁCIDO SULFÚRICO (PT1), PRETRATAMIENTO CON HIDRÓXIDO DE SODIO (PT2), PORCENTAJE DE LIGNINA ÁCIDO SOLUBLE (\% ASL)

\begin{tabular}{|c|c|c|c|c|c|c|c|c|}
\hline \multirow[t]{2}{*}{ Experimento } & \multirow[t]{2}{*}{ PT1 } & \multirow[t]{2}{*}{ PT2 } & \multirow[t]{2}{*}{$\%$ ASL } & \multicolumn{5}{|c|}{ Azúcares reductores $(\mathrm{g} / \mathrm{L})$} \\
\hline & & & & $0.15 \mathrm{~h}$ & $24 \mathrm{~h}$ & $48 \mathrm{~h}$ & $72 \mathrm{~h}$ & $96 \mathrm{~h}$ \\
\hline 1 & -1 & -1 & 4.452 & 0.88 & 0.66 & 1.52 & 1.42 & 0.66 \\
\hline 2 & 0 & -1 & 5.807 & 0.78 & 1.46 & 1.54 & 1.55 & 1.23 \\
\hline 3 & 1 & -1 & 5.935 & 0.94 & 1.30 & 1.29 & 1.42 & 0.68 \\
\hline 4 & -1 & 0 & 8.283 & 0.84 & 1.42 & 0.95 & 0.47 & 0.43 \\
\hline 5 & 0 & 0 & 9.193 & 0.83 & 1.30 & 1.27 & 1.49 & 0.54 \\
\hline 6 & 1 & 0 & 17.522 & 0.88 & 1.26 & 1.29 & 0.63 & 0.49 \\
\hline 7 & -1 & 1 & 14.696 & 0.93 & 1.20 & 1.43 & 0.8 & 0.47 \\
\hline 8 & 0 & 1 & 14.874 & 0.85 & 1.18 & 1.67 & 1.05 & 0.78 \\
\hline 9 & 1 & 1 & 11.453 & 1.01 & 1.22 & 1.32 & 0.62 & 0.50 \\
\hline 10 & 0 & 0 & 9.386 & 0.83 & 1.30 & 1.27 & 1.49 & 0.54 \\
\hline 11 & 0 & 0 & 9.001 & 0.80 & 1.26 & 1.81 & 0.54 & 0.68 \\
\hline 12 & 0 & 0 & 7.401 & 0.78 & 1.46 & 1.54 & 1.55 & 1.23 \\
\hline Testigo & - & - & - & $0.50 \pm 0.21$ & $0.30 \pm 0.01$ & $0.39 \pm 0.01$ & $0.41 \pm 0.07$ & $0.43 \pm 0.01$ \\
\hline
\end{tabular}

Las combinaciones del diseño factorial, los resultados de \% ASL y la cantidad de azúcares reductores liberados en el tiempo se muestran en el cuadro II.

Con los resultados obtenidos, se realizó el análisis estadístico para determinar la significancia de cada variable y su nivel óptimo en la deslignificación del aserrín de pino. Excluyendo los efectos no significativos al $95 \%$ de nivel de confianza $\left(\mathrm{PT}^{2}, \mathrm{PT}^{2}\right.$ y la interacción PT1 x PT2), se obtuvo el diagrama de Pareto que se muestra en la figura 2. Se observó que ambos factores influyen linealmente de manera positiva sobre el sistema y la interacción entre ambos no es significativa. El nivel óptimo de cada factor que favoreció una mayor deslignificación (medida como $\%$ ASL) fue el +1 , que correspondió a $3 \%$ de ácido sulfúrico y $3 \%$ de hidróxido de sodio.

\section{Sacarificación}

En el cuadro III se muestra el análisis de varianza multifactorial (pretratamiento y tiempo de sacarifica-

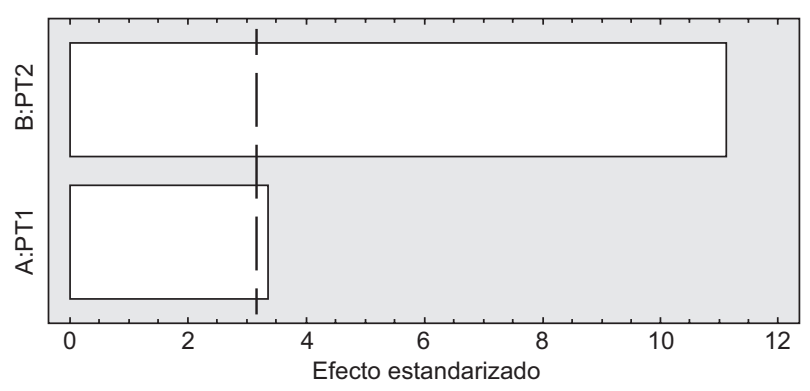

Fig. 2. Diagrama de pareto estandarizado para el \% lignina ácido soluble. $\square$ influye positivamente, $\square$ influye negativamente. estandarización realizada con base en el error estándar. PT1 = pretratamiento con ácido sulfúrico. PT2 $=$ pretratamiento con hidróxido de sodio ción), donde se observó la significancia estadística de los factores, Sin embargo, la interacción entre ellos no fue significativa. Para determinar entre qué niveles de cada factor existieron diferencias, se utilizó la prueba de la diferencia mínima significativa (LSD, por sus siglas en inglés). En el caso del factor pretratamiento, hubo diferencias significativas entre el control y todas las combinaciones de pretratamientos. Sin embargo, entre los pretratamientos, en la mayoría de los casos hubo superposición de medias (Cuadro IV).

Respecto al tiempo de sacarificación, la mayor concentración de azúcares se alcanzó a las 48 h. La prueba LSD para este factor mostró superposición de medias entre las 24 y 48 h. Por lo tanto, la máxima concentración de azúcares reductores se alcanzó en ese lapso de tiempo (Cuadro V).

Dado que la mayor concentración de azúcares reductores se obtuvo entre las 24 y $48 \mathrm{~h}$, se utilizaron ambos tiempos para analizar el diseño (Fig. 3). Eliminando los efectos no significativos $\left(\mathrm{PT}^{2}\right.$ y la interacción PT1 $\times$ $\mathrm{PT} 2$ ), se encontró que el efecto PT $1^{2}$ presentó influencia negativa sobre la concentración de azúcares. Un factor cuadrático negativo y estadísticamente significativo, indica que la variable respuesta (concentración de azúcares) incrementó a medida que el nivel del factor se incrementaba. En tanto que la disminución de la respuesta pudo ser observada cuando el nivel del factor se continuó incrementando a partir de un cierto valor que maximice la respuesta. La concentración de azúcares reductores no se vio afectada por el PT2, pero sí por el PT1, alcanzando un máximo para el nivel 0 del mismo (2\% de ácido sulfúrico). El nivel óptimo de cada factor que favoreció la sacarificación enzimática coincidió con los puntos centrales del experimento, esto es $2 \%$ de ácido sulfúrico y $2 \%$ de hidróxido de sodio. 
CUADRO III. ANÁLISIS DE VARIANZA PARA LOS EFECTOS PRETRATAMIENTO (A) Y TIEMPO DE SACARIFICACIÓN (B)

\begin{tabular}{lccccc}
\hline Efecto & $\begin{array}{c}\text { Suma de } \\
\text { cuadrados }\end{array}$ & $\begin{array}{c}\text { Grados de } \\
\text { libertad }\end{array}$ & $\begin{array}{c}\text { Cuadrado } \\
\text { medio }\end{array}$ & Razón-F & p-valor \\
\hline A & 0.046 & 9 & 0.005 & 7.53 & 0.0001 \\
B & 0.029 & 4 & 0.007 & 10.59 & 0.0001 \\
A x B & 0.027 & 36 & 0.001 & 1.09 & 0.4316 \\
Residuos & 0.013 & 20 & 0.001 & - & - \\
Total & 0.119 & 69 & - & - & - \\
\hline
\end{tabular}

${ }^{a}$ Permite establecer si existen diferencias entre las varianzas de dos grupos de datos. Razón-F = 1 las varianzas coinciden.

\section{CUADRO IV. PRUEBA DE LA MÍNIMA DIFERENCIA SIGNIFICATIVA PARA LOS AZÚCARES REDUCTORES OBTENIDOS POR PRETRA- TAMIENTO REALIZADO}

\begin{tabular}{lcc}
\hline Pretratamiento & Media LS & Grupos homogéneos \\
\hline Control & 0.407 & $\mathrm{X}$ \\
-1 (PT1) 0 (PT2) & 0.823 & $\mathrm{X}$ \\
1 (PT1) 0 (PT2) & 0.910 & $\mathrm{XX}$ \\
1 (PT1) 1 (PT2) & 0.934 & $\mathrm{XX}$ \\
-1 (PT1) 1 (PT2) & 0.966 & $\mathrm{XX}$ \\
-1 (PT1) -1 (PT2) & 1.028 & $\mathrm{XXX}$ \\
0 (PT1) 1 (PT2) & 1.106 & $\mathrm{XXX}$ \\
1 (PT1) -1 (PT2) & 1.125 & $\mathrm{XXX}$ \\
0 (PT1) 0 (PT2) & 1.126 & $\mathrm{XX}$ \\
0 (PT1) -1 (PT2) & 1.312 & $\mathrm{X}$ \\
\hline
\end{tabular}

PT1 = pretratamiento ácido, PT2 = pretratamiento básico, LS = media calculada mediante la prueba de la diferencia mínima significativa

\section{DISCUSIÓN}

\section{Composición química}

El material lignocelulósico de madera de conífera está compuesto aproximadamente por un $40 \%$ de celulosa, $21 \%$ de hemicelulosa y $28 \%$ de lignina (Galbe y Zacchi 2002). Estos resultados concuerdan

CUADRO V. PRUEBA DE LA MÍNIMA DIFERENCIA SIGNIFICATIVA PARA LOS AZÚCARES REDUCTORES OBTENIDOS POR TIEMPO DE SACARIFICACIÓN

\begin{tabular}{ccc}
\hline Tiempo (h) & Media LS & Grupos homogéneos \\
\hline 96 & 0.642 & $\mathrm{X}$ \\
0.15 & 0.842 & $\mathrm{XX}$ \\
72 & 0.964 & $\mathrm{XX}$ \\
24 & 1.133 & $\mathrm{XX}$ \\
48 & 1.288 & $\mathrm{X}$ \\
\hline
\end{tabular}

$\mathrm{LS}=$ media calculada mediante la prueba de la diferencia mínima significativa con los presentados en este trabajo y por otros autores, como López-Miranda et al. (2009), para aserrín de pino. Las diferencias en algunos de los componentes pueden ser estacionales o deberse a diferencias entre especies. El contenido de carbohidratos (65\%) se aproximó a lo obtenido para Pinus taeda (64\%), $P$. contorta $(67 \%)$ y $P$. patula (62\%). Sin embargo, el contenido total de lignina (24\%) fue menor que el obtenido para las citadas especies, variando entre $27 \%$ para $P$. contorta hasta $30 \%$ para $P$. taeda (Ewanick et al. 2007, Gómez et al. 2013, Sannigrahi et al. 2010).

\section{Pretratamiento}

La lignocelulosa de coníferas se considera particularmente recalcitrante hacia la hidrólisis debido a la cantidad y naturaleza de su lignina (Pan et al. 2005). El pretratamiento es fundamental para permitir un mejor proceso de hidrólisis y fermentación a la hora de utilizar biomasa lignocelulósica en la producción de bioetanol (Abril y Abril 2009). Además, el proceso de pretratamiento secuencial conduce a mejorar la eficiencia consumiendo menos químicos, lo que resulta en costos más bajos y menor generación de residuos (Weerasai et al. 2014). La eliminación de lignina modifica la estructura de la madera creando poros o aumentando el tamaño de los ya existentes, lo que produce una organización más abierta y accesible a la penetración de enzimas en la etapa de sacarificación.

Camesasca et al. (2015) obtuvieron $0.024 \mathrm{~g}$ glucosa/g pasto y bajos niveles de subproductos inhibidores con ácido sulfúrico a $1.5 \%$ durante $60 \mathrm{~min}$ a $121{ }^{\circ} \mathrm{C}$. Este valor es bajo comparado con el reportado en este trabajo $(1.81 \mathrm{~g} / \mathrm{L}$ corresponde a $0.077 \pm$ $0.014 \mathrm{~g}$ azúcares/g aserrín en los puntos centrales del diseño, según la cantidad de material sacarificado). Ma et al. (2010) encontraron que, aumentando el tiempo y la temperatura del pretratamiento con ácido sulfúrico diluido, se incrementa el rendimiento en 


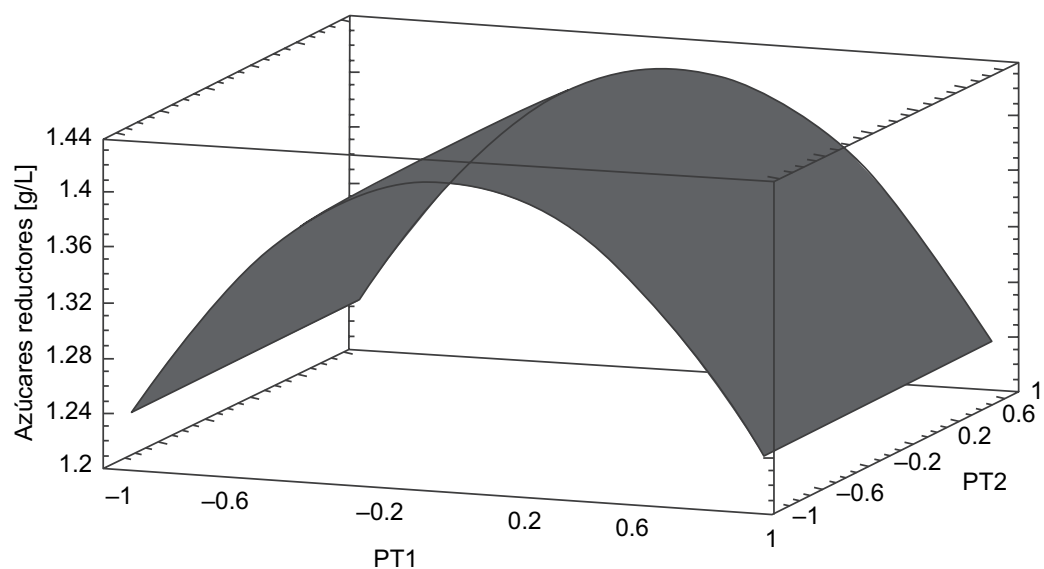

Fig 3. Superficie de respuesta estimada. $\mathrm{PT} 1=$ pretratamiento ácido, $\mathrm{PT} 2=$ pretratamiento básico

azúcares reductores en la hidrólisis enzimática. Sin embargo, lo anterior puede ocasionar un aumento en la concentración de subproductos inhibidores del proceso fermentativo como furfural y 5-hidroximetilfurfural (Saha et al. 2005, Camesasca et al. 2015, Martínez et al. 2015,).

El contenido de lignina soluble se midió en el licor resultante del PT2 debido a que la lignina se solubiliza en solución alcalina acuosa (Jagtap et al. 2013). Las condiciones que generaron mayor pérdida de lignina corresponden al $3 \%$ de $\mathrm{NaOH}(57.07 \%$ calculado a partir del \% ASL). Hosgun y Bozan (2014) obtuvieron un valor similar de remoción de lignina (58.89\%) con hidróxido de sodio al $2.25 \%$ utilizando tallos de Papaver somniferum. Silverstein et al. (2007) hallaron que el pretratamiento con ácido sulfúrico a $121^{\circ} \mathrm{C}$ fue más eficaz para la solubilización de xilano y el pretratamiento con hidróxido de sodio produjo el $66 \%$ de pérdida de lignina al $2 \%$ por 90 min a 121 ${ }^{\circ} \mathrm{C}$. En este último caso, la mayor pérdida de lignina puede deberse a las diferencias en el tipo de material utilizado. En concentraciones menores de ácido (1\%) y álcali (1.25\%), Weerasai et al. (2014) obtuvieron una pérdida de lignina del $45.69 \%$ utilizando paja de arroz.

\section{Sacarificación}

El principal desafío tecno-económico en todo proceso de bioconversión de lignocelulosa a bioetanol es superar la fracción de celulosa recalcitrante ante la hidrólisis enzimática. La celulosa es intrínsecamente resistente al ataque enzimático y además está protegida por la matriz envolvente de lignina y hemicelulosa (Pan et al. 2005).

Para degradar biológicamente la celulosa son necesarias distintas enzimas con diferentes especificidades trabajando juntas: endoglucanasas y celobiohidrolasas que producen celobiosa como producto final, y $\beta$-glucosidasas que rompen la celobiosa y liberan dos moléculas de glucosa (Pérez et al. 2002).

El valor máximo de azúcares reductores obtenidos $(1.81 \mathrm{~g} / \mathrm{L})$ fue comparable al obtenido por Jeya et al. (2010) con biomasa leñosa y una carga enzimática de 20 unidades de papel de filtro, (FPU, por sus siglas en inglés)/g sustrato (2.9 g azúcares/L a las $50 \mathrm{~h}$ de sacarificación). Yamashita et al. (2010) no observaron producción de glucosa y azúcares reductores en la sacarificación enzimática del bambú sin pretratar. Sin embargo, en el presente trabajo el aserrín de pino sin pretratar (testigo) produjo azúcares reductores al ser sacarificado $(0.43 \mathrm{~g} / \mathrm{L}$ a las $96 \mathrm{~h})$. No obstante, existieron diferencias estadísticamente significativas entre los azúcares obtenidos entre el material pretratado y el material sin pretratar.

Respecto al tiempo de sacarificación, la mayor concentración de azúcares se obtuvo entre las 24 - 48 $\mathrm{h}$ (1.21 g/L en promedio), datos que coinciden con lo reportado por otros autores (Dogaris et al. 2009, López-Miranda et al. 2009, Jeya et al. 2010).

\section{CONCLUSIONES}

Se obtuvo una elevada deslignificación del aserrín de pino que se correspondió con las mayores concentraciones de ácido y álcali empleadas en el trabajo (3\% de cada una). Se optimizaron las concentraciones de ácido y álcali del pretratamiento secuencial de aserrín de pino, respecto a la producción, por hidrólisis enzimática de su contenido de celulosa en azúcares reductores, siendo éstos cuatro veces mayores que el aserrín sin pretratar. Esto evidencia que la mayor eliminación de lignina en el pretratamiento, condujo 
a una mejor acción de las enzimas en la sacarificación. La cantidad de azúcares reductores obtenidos fue comparable a lo obtenido por otros autores. Sin embargo, es posible alcanzar mejores resultados optimizando además la etapa de sacarificación, al ajustar por ejemplo, la carga enzimática respecto del material pretratado.

\section{AGRADECIMIENTOS}

Este trabajo fue financiado por el Proyecto de Investigación Científico-Tecnológico Orientado y la Universidad Nacional de Misiones 2011-0086, Agencia Nacional de Promoción Científica y Tecnológica, Ministerio de Ciencia, Tecnología e Innovación Productiva, República Argentina. Los autores desean agradecer el financiamiento otorgado por la Secretaría General de Ciencia y Tecnología de la Universidad Nacional de Misiones (16Q446). María Daniela Rodríguez, María Lorena Castrillo, Juan Ernesto Velázquez y Gustavo Raúl Kramer poseen becas del Consejo Nacional de Investigaciones Científicas y Técnicas (CONICET), Argentina.

\section{REFERENCIAS}

Abril D. y Abril A. (2009). Ethanol from lignocellulosic biomass. Cien. Inv. Agr. 36 (2), 177-190. DOI: $10.4067 / \mathrm{S} 0718-16202009000200003$

Brodeur G., Yau E., Badal K., Collier J., Ramachandran K.B. y Ramakrishnan S. (2011). Chemical and physicochemical pretreatment of lignocellulosic biomass: A review. Enzyme Research 2011, 1-17. DOI: $10.4061 / 2011 / 787532$

Camesasca L., Ramírez M. B., Guigou M., Ferrari M.D. y Lareo C. (2015). Evaluation of dilute acid and alkaline pretreatments, enzymatic hydrolysis and fermentation of napiergrass for fuel ethanol production. Biomass Bioenerg. 74, 193-201.

DOI: 10.1016/j.biombioe.2015.01.017

Carvalheiro F., Duarte L.C. y Gírio F.M. (2008). Hemicellulose biorefineries: A review on biomass pretreatments. J. Sci. Ind. Res. 67, 849-864.

Dogaris I., Vakontios G., Kalogeris E., Mamma D. y Kekos D. (2009). Induction of cellulases and hemicellulases from Neurospora crassa under solid-state cultivation for bioconversion of sorghum bagasse into ethanol. Ind. Crop. Prod. 29 (2-3), 404-411.

DOI: $10.1016 /$ j.indcrop.2008.07.008

Ewanick S.M., Bura R. y Saddler J.N. (2007). Acidcatalyzed steam pretreatment of lodgepole pine and subsequent enzymatic hydrolysis and fermentation to ethanol. Biotechnol. Bioeng. 98 (4), 737-746.

DOI: $10.1002 /$ bit.21436

Galbe M. y Zacchi G. (2002). A review of the production of ethanol from softwood. Appl. Microbiol. Biotechnol. 59 (6), 618-628. DOI: 10.1007/s00253-002-1058-9

Gómez E.A., Ríos L.A. y Peña J.D. (2013). Efecto del pretratamiento de biomasa maderera en el rendimiento a etanol. Información Tecnológica 24 (5), 113-122. DOI: $10.4067 / \mathrm{S} 0718-07642013000500013$

Hosgun E.F. y Bozan B. (2014). Comparison of high temperature alkali and acid pretreatment for the enzymatic saccharification of Poppy stalks. Chem. Eng. Trans. 37, 373-378. DOI: 10.3303/CET1437063

Jagtap S.S., Dhiman S.S., Kim T., Li J., Lee J. y Kang Y.C. (2013). Enzymatic hydrolysis of aspen biomass into fermentable sugars by using lignocellulases from Armillariagemina. Bioresource Technol. 133, 307-314. DOI: 10.1016/j.biortech.2013.01.118

Jeya M., Nguyen N., Moon H., Kim S. y Lee J. (2010). Conversion of woody biomass into fermentable sugars by cellulase from Agaricusarvensis. Bioresource Technol. 101 (22), 8742-8749.

DOI: 10.1016/j.biortech.2010.06.055

Lin Y. y Tanaka S. (2006). Ethanol fermentation from biomass resources: current state and prospects. Appl. Microbiol. Biotechnol. 69 (6), 627-642.

DOI: $10.1007 / \mathrm{s} 00253-005-0229-\mathrm{x}$

López-Miranda J., Soto-Cruz N., Rutiaga-Quiñones O.M., Medrano-Roldán H. y Arévalo-Niño K. (2009). Optimización del proceso de obtención enzimática de azúcares fermentables a partir de aserrín de pino. Rev. Int. Contam. Ambie. 25 (2), 95-102.

Ma F., Yang N., Xu C., Yu H., Wu J. y Zhang X. (2010). Combination of biological pretreatment with mild acid pretreatment for enzymatic hydrolysis and ethanol production from water hyacinth. Bioresource Technol. 101 (24), 9600-9604.

DOI: 10.1016/j.biortech.2010.07.084

Mantulak M.J., Michalus J.C., Hernández Pérez G., Dekun M.C. y Kerkhoff A.F. (2013). Gestión de los residuos tecnológicos en pequeños aserraderos, Misiones, Argentina. Memorias. Salao do Conhecimento, XVIII Jornada de Pesquisa, UNIJUI, Ijuí, Santa Rosa, Brasil. Del 10 al 13 de septiembre, CD-ROM.

Miller G.L. (1959). Use of dinitrosalicylic acid reagent for determination of reducing sugar. Anal. Chem. 31 (3), 426-428. DOI: $10.1021 / \mathrm{ac} 60147 \mathrm{a} 030$

Murciano Martínez P., Bakker R., Harmsen P., Gruppen H. y Kabel M. (2015). Importance of acid or alkali concentration on the removal of xylanand lignin for enzymatic cellulose hydrolysis. Ind. Crop. Prod. 64, 88-96. DOI: 10.1016/j.indcrop.2014.10.031 
NREL (2008a). NREL/TP-510-42619, Determination of extractives in biomass. National Renewable Energy Laboratory. United States Department of Energy. Laboratory Analytical Procedures. Enero de 2008.

NREL (2008b). NREL/TP-510-42628, Measurement of cellulase activities. National Renewable Energy Laboratory. United States Department of Energy. Laboratory Analytical Procedures. Enero de 2008.

NREL (2008c). NREL/TP-510-42622, Determination of ash in biomass. National Renewable Energy Laboratory. United States Department of Energy. Laboratory Analytical Procedures. Enero de 2008.

NREL (2008d). NREL/TP-510-42621, Determination of total solids in biomass and total dissolved solids in liquid process samples. National Renewable Energy Laboratory. United States Department of Energy. Laboratory Analytical Procedures. 31 de marzo de 2008.

NREL (2011). NREL/TP-510-42618, Determination of structural carbohydrates and lignin in biomass. National Renewable Energy Laboratory. United States Department of Energy. Laboratory Analytical Procedures. 7 de agosto de 2011.

Pan X., Xie D., Gilkes N., Gregg D.J. y Saddler J.N. (2005). Strategies to enhance the enzymatic hydrolysis of pretreated softwood with high residual lignin content. Simposio. Twenty-Sixth Symposium on Biotechnology for Fuels and Chemicals. Applied Biochemistry and Biotechnology Symposium. Chattanooga, Tennessee, Estados Unidos. 9 al 12 de mayo de 2004, pp. 1069-1079. DOI: 10.1007/978-1-59259-991-2

Pérez J., Muñoz-Dorado J., de la Rubia T. y Martínez J. (2002). Biodegradation and biological treatments of cellulose, hemicellulose and lignin: an overview. Int. Microbiol. 5 (2), 53-63.

DOI: $10.1007 / \mathrm{s} 10123-002-0062-3$
Romero R.B. (2014). Energías alternativas - Biomasa forestal. Memorias. IX Congreso argentino de derecho agrario. Buenos Aires, Argentina 28 y 29 de mayo de 2014. CD-ROM.

Saha B.C.,Iten L.B., Cotta M.A. y Wu Y.V. (2005). Dilute acid pretreatment, enzymatic saccharification and fermentation of wheat straw to etanol. Biotechnol. Progr. 21 (3), 816-822.

DOI: $10.1021 / \mathrm{bp} 049564 \mathrm{n}$

Sánchez A., Gil J.C., Rojas-Rejón O.A., de Alba A.P., Medina A., Flores R. y Puente R. (2015). Sequential pretreatment strategies under mild conditions for efficient enzymatic hydrolysis of wheat straw. Bioprocess Biosyst. Eng. 38 (6), 1127-1141. DOI: $10.1007 / \mathrm{s} 00449-015-1355-1$

Sannigrahi P., Miller S.J. y Ragauskas A.J. (2010). Effects of organosolv pretreatment and enzymatic hydrolysis on cellulose structure and crystallinity in Loblolly pine. Carbohyd Res. 345 (7), 965-970. DOI: 10.1016/j.carres.2010.02.010

Silverstein R.A., Chen Y., Sharma-Shivappa R.R., Boyette M.D. y Osborne J. (2007). A comparison of chemical pretreatment methods for improving saccharification of cotton stalks. Bioresource Technol. 98 (16), 3000-3011.

DOI: $10.1016 /$ j.biortech.2006.10.022

Weerasai K., Suriyachai N., Poonsrisawat A., Arnthong J., Unrean P., Laosiripojana N. y Champreda V. (2014). Secuential acid and alkaline pretreatment of rice straw for bioetanol fermentation. Bioresources 9 (4), 5988-6001.

Yamashita Y., Shono M., Sasaki C. y Nakamura Y. (2010). Alkaline peroxide pretreatment for efficient enzymatic saccharification of bamboo. Carbohyd. Polym. 79 (4), 914-920. DOI: 10.1016/j.carbpol.2009.10.017 